been conspicuously associated with these outstanding achievements. Various chain-lengthening methods, which have played an important part in the synthetic investigations, are prominently featured and stereochemical considerations receive due attention.

The last chapter deals with the oxidative coupling of acetylenes through the intermediacy of the cuprous derivatives. Discovered by Glaser some 90 years ago and applied by Baeyer in $\mathbf{1 8 8 2}$ in his historic synthesis of indigo, this reaction, in its various modifications, has proved of great utility. It has figured in the synthesis of naturally occurring polyacetylenes and has been elegantly applied for the synthesis of a whole range of novel, unsaturated macrocycles including the first allplanar, cyclic homologue of benzene, [18]-annulene. The full scope and versatility of this reaction are clearly demonstrated in this review. However, one is left with the impression that much remains to be learned concerning its mechanistic aspects. While it has been brilliantly exploited by the organic chemists for preparative purposes it has been very much neglected by physical chemists and co-ordination chemists. Maybe this critical appraisal of the present state of knowledge will inspire fresh endeav. ours to shed more light on the course of these acetylene coupling reactions and the not unrelated oxidative coupling of phenols and aromatic amines by the same reagents.

The declared motto of one of the reviews is "to stimulate rather than to satisfy". Every single contribution will certainly not fail to stimulate and most, if not all, organic chemists will feel more than satisfied with the wealth of well-documented and systematically presented information which has been crystallized within these pages.

J. IDRIS JONES

\section{MÖSSBAUER SPECTRA AND CHEMICAL STRUCTURE}

The Mössbauer Effect and Its Applications in Chemistry By V. I. Gol'danskii. Authorized translation from the Russian. Pp. vii + 119. (New York: Consultants Bureau, 1964.) 12.50 dollars.

$\mathrm{T}$ HE effect of recoil-less emission and resonant reabsorption of $\gamma$-rays was first reported by Rudolf Mössbauer in 1958 and it has already been used for a wide variety of elegant experiments in basic physics. During the past three years it has been increasingly recognized that the Mössbauer effect can also give valuable information on chemical problems, and one of the pioneers in this field, Prof. V. I. Gol'danskii, has now written the first monograph specifically aimed at describing these chemical applications. The treatment throughout is nonmathematical and eminently readable.

The account begins with a brief discussion of the energies and widths of resonance transitions. When an excited atom is bound in a crystal the $\gamma$-ray may be emitted without recoil or thermal broadening and the precision with which its energy is then defined depends only on the Heisenberg uncertainty relation. Energy quanta of unprecedented precision are therefore available and these have been used to probe the minute changes in the separation of the nuclear energy-levels which result from differing chemical environments. These ideas are developed in the second chapter, which also mentions briefly the appropriate experimental techniques.

In any spectroscopic technique, information can be derived from the number of lines observed, from their position, shape and intensity. In Mössbauer spectroscopy these parameters give information about chemical shifts, quadrupole coupling constants and hyperfine magnetic fields. The origin of these effects is traced in Chapters 3 and 4.
The Mössbauer effect has been detected in a large number of elements with atomic numbers greater than 26 and these elements are spread widely throughout the Periodic Table. The most extensively investigated nuclide is ${ }^{57} \mathrm{Fe}$ and results on the compounds of iron are reviewed in Chapter 5. Alloys, mixed oxides, salts, co-ordination complexes and organometallic compounds are mentioned.

Compounds of tin have also been widely investigated and the results are discussed in Chapter 6. This is followed by an assessment of various other problems which might be tackled by Mössbauer spectroscopy. There is an appendix listing the relevant properties of some eighty nuclides of the forty-five elements for which resonance has been observed or is predicted, and the review ends with a supplement outlining results published during 1963.

Prof. Gol'danskii has provided us with a persuasively written and exciting account of this new technique and it is unfortunate that his efforts have not been matched by those of the publishers. The translation, though generally good, is marred by occasional oddities like "gamma transactions", "very weakly pronounced" and "defectoscopy". The typesetting is also variable in quality and it is difficult in places to distinguish between $\Gamma, \gamma$, and $r$. The binding is inadequate and the price very high. The original Russian publication cost about 30 cents and an English translation is already available from the International Atomic Energy Agency, in Vienna. There is a further disturbing feature: this is a 65-page review which is being made by the publishers to masquerade as a 120-page book. There is no indication from the title on the cover, from the fly-leaf, from the title page itself, or from the author's preface that half the book has nothing to do with the Mössbauer effect but is a translation of a four-year-old paper on another subject by other authors, whose names do not even appear in the table of contents.

N. N. GREENWOOD

\section{WIDENING FIELD OF QUANTUM ELECTRONICS}

\section{Quantum Electronics}

Proceedings of the Third International Congress, Paris. Edited by Prof. P. Grivet and Prof. N. Bloembergen. Vol. 1: pp. xxix +1-966. Vol. 2: pp. 967-1923. (Paris: Dunod, New York and London: Columbia University Press, 1964.) 252s. the two volumes.

$\mathrm{Q}$ UANTUM electronics is a term which was originally coined to cover the investigation of resonant phenomena associated with the interaction of radiation and matter. With the growth of new techniques has come an inevitable spreading of the boundaries of the subject. Some of the topics are new, such as non-linear optical effects and the stimulated Raman effect; in others interest has been revived. The development of the maser is responsible for a great part of this growth, particularly in its optical form, the laser.

The third international conference on "Quantum Electronics" was held in Paris in February 1963. (The term 'Congress' is used only on the title page of the Proceedings.) There were some 1,100 participants, compared with 160 at the first conference in September 1959 , and 450 at the second in March 1961, ample evidence of the growth of interest in the subject. In all probability this will be the last conference of this nature; no suitable accommodation is available, for the numbers that are likely to wish to attend in future.

There has been a long delay in the publication of the Proceedings of the conference, but the result has been well worth the wait. Two hundred of the papers presented at the conference are published, thirty-two of them in French, the rest in English. Among the new topics that 